\title{
headspace - Australia's innovation in youth mental health: who are the clients and why are they presenting?
}

\section{Debra J Rickwood \\ BA(Hons), PhD, FAPS Professor of Psychology, and Chief Scientifi Advisor $^{2}$ \\ Nic R Telford BSS, MSS Evaluation Manager \\ Alexandra G Parker BA(Hons) \\ MClinPsych, PhD, \\ Director, headspace Centre of Excellence \\ Chris J Tanti BA, BSW, AMP \\ CEO \\ Patrick D McGorry MD, PhD, FRANZCP \\ Professor of Youth \\ Mental Health \\ 1 Faculty of Health, University of Canberra \\ Canberra, ACT. \\ 2 headspace National Youth Mental Health \\ Foundation, \\ Melbourne, VIC \\ 3 Orygen Youth Health Research Centre \\ University of Melbourne \\ Melbourne, VIC. \\ debra.rickwood@ \\ canberra.edu.au}

MJA 2014:200: 1-4 doi: 10.5694/mjal3.11235

Online first 13/01/14 eadspace National Youth Mental Health Foundation is the

Australian Government's major investment in the area of youth mental health. ${ }^{1}$ The National Survey of Mental Health and Wellbeing (NSMHW) revealed that one in four young people experience a clinically relevant mental health problem within any 12-month period, compared with one in five in the general population. $^{2}$ Half of a cohort of young people were shown to suffer diagnosable mental ill health at some point during the transition from childhood to adulthood, which reduces fulfilment of their potential and increases likelihood of disability and premature death. ${ }^{3}$ Australian data are consistent with international trends and the adolescent and early adult years are periods of peak prevalence and incidence for most mental disorders. ${ }^{4,5}$ Yet, despite having the highest prevalence, young people have the lowest level of professional help-seeking for mental health problems across the lifespan. $^{2}$

headspace was initiated in 2006 to address the concerning mismatch between level of need and amount of mental health service use among adolescents and young adults. ${ }^{1}$ The initiative is innovative in targeting the age range from early adolescence through early adulthood, maintaining that the traditional child and adolescent versus adult service divide creates a disjunction at precisely the time when there is greatest need for continuity. ${ }^{6}$ There are now 55 headspace centres across Australia, scaling up to 100 centres in 2016.

The approach to service delivery has been described elsewhere, ${ }^{1,7}$ but briefly, headspace centres aim to create highly accessible, youth-friendly, integrated service hubs that provide evidence-based interventions and support to young people aged 12-25 years around their mental health, health and wellbeing needs. Each centre is directed by a lead agency on

Objectives: To provide the first national profile of the characteristics of young people (aged 12-25 years) accessing headspace centre services - the Australian Government's innovation in youth mental health service delivery - and investigate whether headspace is providing early service access for adolescents and young adults with emerging mental health problems.

Design and participants: Census of all young people accessing a headspace centre across the national network of 55 centres comprising a total of 21274 headspace clients between 1 January and 30 June 2013.

Main outcome measures: Reason for presentation, Kessler Psychological Distress Scale, stage of illness, diagnosis, functioning.

Results: Young people were most likely to present with mood and anxiety symptoms and disorders, self-reporting their reason for attendance as problems with how they felt. Client demographic characteristics tended to reflect population-level distributions, although clients from regional areas and of Aboriginal and Torres Strait Islander background were particularly well represented, whereas those who were born outside Australia were underrepresented.

Conclusion: headspace centres are providing a point of service access for young Australians with high levels of psychological distress and need for care in the early stages of the development of mental disorder.

behalf of a local partnership of organisations responsible for the delivery of services, comprising mental health, alcohol and other drug, primary care, and vocational services. The main aim is to improve outcomes for young people by addressing the major barriers to service use for young people, ${ }^{8,9}$ and enabling better access to and engagement in early intervention services that provide holistic and integrated care.

The current study provides the first comprehensive profile of headspace clients across the entire national network of the current 55 centres. It is timely to investigate the demographic characteristics of young people presenting to headspace centres and their reasons for presentation to determine whether headspace is providing early service access for adolescents and young adults with emerging mental health problems.

\section{Method}

\section{Participants and procedure}

Participants were all headspace clients who received a centre-based service between 1 January and 30 June 2013. This comprised data from 21274 clients across the 55 current headspace centres. The centres have been operational for varying periods of time, including 10 round 1 centres (established in 2007), 20 round 2 centres (2009), 10 round 3 centres (2011), and 15 round 4 centres established in the past 12 months.

A major review in 2012 of routine data collected by headspace found that beyond basic demographics, the information was generally of poor quality. Consequently, a new minimum dataset was implemented from the beginning of 2013. This requires young people accessing headspace centres and their service providers to enter data into an electronic form about each occasion of service. Data are de-identified by encryption and extracted to the headspace national office data warehouse.

\section{Measures}

Client demographic characteristics comprised age in years, sex, Aboriginal and Torres Strait Islander background, country of birth, living situation and current occupation. 


\begin{tabular}{|c|c|c|c|c|c|c|c|c|}
\hline & \multicolumn{4}{|c|}{ Male } & \multicolumn{4}{|c|}{ Female } \\
\hline & $12-14$ years & $15-17$ years & $18-20$ years & $21-25$ years & $12-14$ years & $15-17$ years & $18-20$ years & $21-25$ years \\
\hline $\begin{array}{l}\text { Proportion of total male or } \\
\text { female clients }\end{array}$ & $13.4 \%$ & $30.4 \%$ & $27.3 \%$ & $28.9 \%$ & $11.5 \%$ & $36.8 \%$ & $27.1 \%$ & $24.7 \%$ \\
\hline \multicolumn{9}{|l|}{ Reason for presenting } \\
\hline Feelings & $62.3 \%$ & $66.7 \%$ & $73.8 \%$ & $76.3 \%$ & $67.5 \%$ & $71.9 \%$ & $70.2 \%$ & $72.6 \%$ \\
\hline Relationships & $19.6 \%$ & $14.1 \%$ & $9.3 \%$ & $8.0 \%$ & $19.6 \%$ & $13.3 \%$ & $8.7 \%$ & $7.8 \%$ \\
\hline School/work & $16.2 \%$ & $10.2 \%$ & $4.2 \%$ & $3.9 \%$ & $9.9 \%$ & $6.2 \%$ & $3.4 \%$ & $4.0 \%$ \\
\hline Physical health & $1.1 \%$ & $3.4 \%$ & $4.6 \%$ & $5.5 \%$ & $2.4 \%$ & $5.2 \%$ & $12.0 \%$ & $11.8 \%$ \\
\hline Alcohol/drugs & $0.6 \%$ & $3.6 \%$ & $4.8 \%$ & $4.3 \%$ & $0.2 \%$ & $0.5 \%$ & $0.9 \%$ & $1.1 \%$ \\
\hline Vocational & $0.1 \%$ & $1.6 \%$ & $3.1 \%$ & $1.9 \%$ & $0.1 \%$ & $0.4 \%$ & $0.9 \%$ & $0.6 \%$ \\
\hline Sexual health & 0 & $0.4 \%$ & $0.3 \%$ & $0.1 \%$ & $0.3 \%$ & $2.6 \%$ & $3.9 \%$ & $1.6 \%$ \\
\hline
\end{tabular}

Client clinical presentation characteristics were measured through selfreported reason for presentation, as well as by clinician diagnosis according to relevant World Health Organization ICD-10 classifications of mental and behavioural disorders. Level of psychological distress was measured by self-report using the 10item Kessler Psychological Distress Scale (K10), ${ }^{10}$ while stage of illness was estimated by clinicians using the categories of no mental disorder, mild to moderate symptoms, subthreshold symptoms not reaching full diagnosis, diagnosed disorder, periods of remission, or serious and ongoing disorder without periods of remission. ${ }^{11}$ Days out of role were self-reported, ${ }^{12}$ and overall functioning was assessed by clinicians using the Social and Occupational Functioning Assessment Scale (SOFAS). ${ }^{13}$

\section{Results}

\section{Client demographic characteristics}

The proportion of male and female clients in each age group is shown in Box 1 . The peak age of presentation was 15-17 years, and relatively more males presented in the youngest (1214 years) and oldest (21-25 years) age groups. Overall, $63.7 \%$ of clients were female and $35.6 \%$ were male, with only $0.7 \%$ reporting that they were intersex, transgender or transsexual. The NSMHW showed that $30 \%$ of young women and $23 \%$ of young men had experienced mental disorder in the past 12 months. ${ }^{14}$

There were $7.7 \%$ of clients who identified as Aboriginal or Torres Strait Islander, compared with 2011 census data showing that $4.0 \%$ of Australians aged $12-25$ years identify as Aboriginal or Torres Strait Islander. $^{15}$

Clients who reported being born outside Australia comprised 7\%, compared with $15 \%$ of the population aged 10-24 years in 2007-2008. ${ }^{16}$ Consistent with population trends, the most common places of birth outside Australia for headspace clients were England and New Zealand. Ninety-four per cent reported speaking only English at home, which compares with $80.3 \%$ in the general population aged over 5 years. ${ }^{17}$

Over half the clients $(57.1 \%)$ lived in major cities, while $31.2 \%$ lived in inner regional areas, $9.6 \%$ in outer regional, and $2.1 \%$ in remote or very remote areas. This compares with 2012 estimates from the Australian Bureau of Statistics that $70 \%$ of the youth population lived in major cities, $18 \%$ in inner regional areas, $9 \%$ in outer regional and $2 \%$ in remote or very remote areas. ${ }^{18}$

Most headspace clients had stable accommodation (86.6\%), but there were $10.3 \%$ for whom accommodation was an issue, $2.4 \%$ who reported that they were at risk of being homeless, and $0.7 \%$ who were currently homeless. This compares to $0.7 \%$ of the Australian population aged 12-24 years who were estimated as being homeless or in marginal housing in the 2011 census. ${ }^{19}$ Security of housing decreased markedly with age among headspace clients, from $94.0 \%$ of those aged $12-14$ years to $81.5 \%$ of those aged 21-25 years.

Many clients were currently engaged in education, with $46.7 \%$ at school and $21.0 \%$ in higher education.
Among those aged 18-25 years, 29.0\% were not engaged in employment, education or training, which compares with $27.3 \%$ in the population. ${ }^{20}$

\section{Presentation characteristics}

Overwhelmingly, the main selfreported reason for young people presenting at headspace centres was having problems with how they felt $(71.6 \%)$; specifically, almost a quarter first presented feeling sad or depressed $(24.9 \%)$ and $12.7 \%$ were feeling anxious. The next most common reason was having relationship problems $(11.4 \%)$, followed by physical health issues $(6.6 \%)$, school/work problems $(6.0 \%)$, alcohol or other drug problems $(1.7 \%)$, sexual health issues $(1.6 \%)$ and vocational concerns $(1.0 \%)$. Reasons for presenting varied by age and sex (Box 1). Relationship and school issues decreased with age, while problems with feelings increased, especially for males. For females, health and sexual health reasons for presentation increased with age, while alcohol and other drug and vocational issues become more pressing for males.

Over half $(69.3 \%)$ of the young people attending headspace did so with high or very high levels of psychological distress (Box 2). This compares with only $9 \%$ in the general community aged 16-24 years, and $21 \%$ of young people diagnosed with mental disorder in the NSMHW. ${ }^{14}$ Males aged 12-14 years were most likely to present with the lowest levels of psychological distress, while females aged 15-20 years were most likely to present at the highest level of distress. By early adulthood, the distress levels of males and females con- 
2 Proportion of headspace clients at each level of psychological distress, by age group and sex

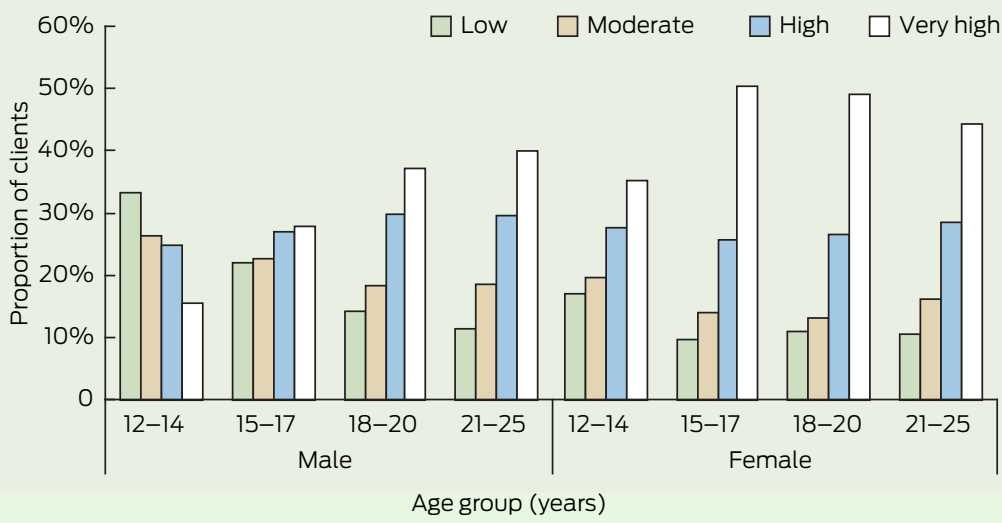

3 Proportion of headspace clients at each stage of illness, by age group and sex

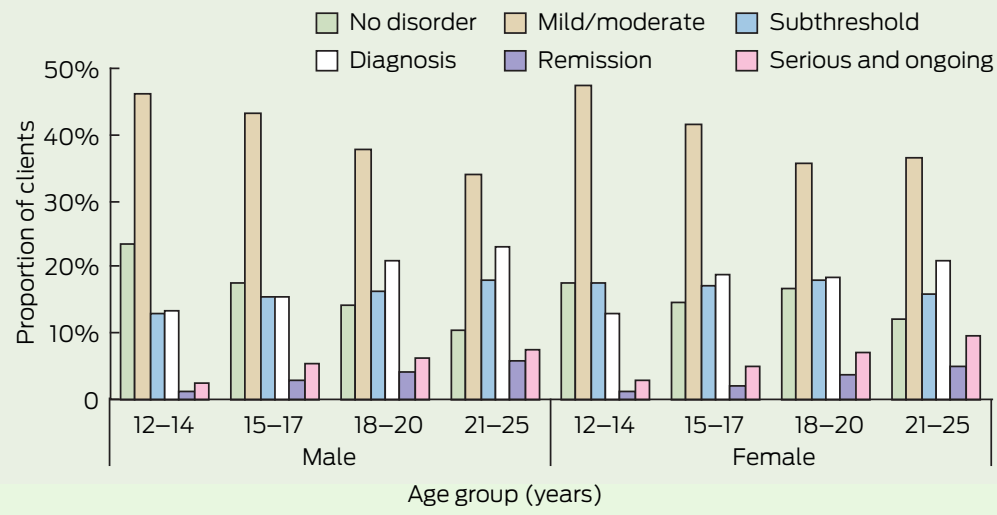

verged. These patterns were reflected in the mean K10 scores. For males, these increased from 20.7 (standard deviation [SD], 8.2) for those aged 12 14 years to 26.9 (SD, 9.0) for those aged 21-25 years. The increase for females was less pronounced, from 25.7 (SD, 9.4) to 27.7 (SD, 9.1) for those aged 12-14 years and 21-25 years, respectively. Only the younger boys had a mean in the moderate range for the K10; means for all other age groups were in the high-distress range.

Stage of illness development reflected the expected age-related trajectory using a population health approach based on the spectrum of mental health interventions ${ }^{21}$ (Box 3). Overall, there were $14.6 \%$ of clients with no mental disorder, $39.6 \%$ with mild to moderate symptoms, $16.9 \%$ with subthreshold diagnosis, $18.8 \%$ with full-threshold diagnosis, 3.5\% with periods of remission, and $6.4 \%$ with serious and ongoing mental disorder. With increasing age, there were fewer clients in the no mental disorder and mild to moderate groups, relative stability in the subthreshold group, and increased proportions in the full-threshold diagnosis, remission, and serious or ongoing disorder categories.

These trends were confirmed by self-report of whether prior mental health care had been received. Overall, a third of clients reported never previously seeing a mental health professional. The proportion declined with age: $51.6 \%$ of $12-14$-year-olds, $41.9 \%$ of $15-17$-year-olds, $31.5 \%$ of $18-20$-year-olds, and $26.4 \%$ of $21-25$ year-olds.

Twenty-nine per cent of clients were estimated by clinicians to have full-threshold, remission, or serious and ongoing disorder, yet almost a third of these had no actual clinical diagnosis recorded at presentation, and a further $6.7 \%$ were reported as diagnosis not yet assessed. The most common diagnoses recorded were mood disorders (28.2\%), followed by anxiety disorders (17.3\%), adjustment disorder $(4.3 \%)$, personality disorders $(2.7 \%)$, developmental disorders $(2.3 \%)$, substance use disorders $(1.8 \%)$, psychotic disorders $(1.6 \%)$ and eating disorders (1.2\%).

Self-reported days out of role in the previous 2 weeks revealed a trend of increasing disability due to mental health problems with age. This was most pronounced for males, who were most likely to report no days out of role when aged 12-14 years $(55.5 \%)$, decreasing to $44.5 \%$ for those aged 21-25 years. For females, this decrease was less pronounced, from $41.2 \%$ for those aged 12-14 years to $38.4 \%$ for $21-25$-year-olds. Overall, $40.6 \%$ of headspace clients reported no days out of role, $22.8 \%$ reported 1-3 days, $17.8 \%$ reported 4 6 days, $6.2 \%$ reported $7-9$ days, and $12.5 \%$ reported more than 10 days out of role in the past fortnight.

Social and vocational functioning, as reported by service providers, showed a similar pattern. The proportions of clients with serious or major impairment (SOFAS scores $<50)$ were $11.2 \%, 11.9 \%, 17.0 \%$ and $19.4 \%$ for males in each of the ascending age groups, respectively; and $8.1 \%, 10.0 \%, 12.3 \%$ and $12.6 \%$ for females. The mean SOFAS scores were similar across age and sex and closest to the anchor defined as "Moderate difficulty in social, occupational, or school functioning (eg, few friends, conflicts with peers or coworkers)".

\section{Discussion}

These are the first data that describe the young people presenting to headspace centres across Australia. Such information is timely, as the initiative is now established and attracting national and international interest. Therefore, it is important to examine whether headspace centres are being accessed by their intended target group.

The results show that almost twothirds of headspace clients are female, which partly reflects the sex difference in the distribution of mood and anxiety disorders for this age group within the Australian population. ${ }^{14}$ However, in the future, headspace will need to respond more effectively to mental ill 
health in young men, which typically manifests through substance misuse and behavioural problems, conditions that can mask underlying emotional disorders. The higher proportion of Aboriginal and Torres Strait Islander clients, compared with their proportional representation in the overall population, indicates the need for mental health support in this population group and that the youth-friendly focus of headspace centres may be attractive to young Aboriginal and Torres Strait Islander people. In contrast, there is a lower proportion of clients born outside Australia compared with their proportional representation in the overall population, which suggests that some of these demographic groups may still experience significant barriers to service use.

That a third of the young adult headspace clients were not engaged in education, employment or training indicates the vulnerability of youth with mental health issues to disengagement from vocational opportunities. This is a situation that must be addressed, particularly during the current period of growing unemployment. $^{22}$

headspace is serving young people outside major metropolitan areas an excellent outcome for Australia, which struggles to provide an effective mental health service response in regional and rural areas. ${ }^{23}$ There has been a deliberate strategy to locate headspace centres in regional areas, with the aim of eventually providing national coverage so that all young people have reasonable access to services.

The presenting issues for young people attending headspace centres are primarily problems with how they feel, mostly related to feeling depressed or anxious. The initiative was set up to better respond to the need for care for such high-prevalence mental ill health in young people. However, formal diagnosis of mental disorder by an appropriately qualified clinician is available for only a small proportion of clients. This partly reflects the multidisciplinary nature of the headspace workforce, many of whom are not trained in formal diagnosis and who use psychotherapeutic approaches that are not diagnosis driven. It also suggests the need for an expanded diagnostic approach that incorporates clinician assessment of at-risk and subthreshold conditions. ${ }^{24}$ Over half the young people presenting were in the early stages of the development of mental disorder, having mild to moderate or subthreshold symptoms, as specifically targeted by the initiative. Nevertheless, almost $20 \%$ had an established disorder and about 10\% had a serious ongoing disorder, showing the wide range of clinical presentations that headspace centres need to accommodate.

This description of the presenting characteristics of young people accessing headspace centres suggests that the initiative is mostly achieving its aim to improve service access early in the development of mental illness, although there are demographic groups where access needs to be improved. Further analyses of the new minimum dataset are planned, to examine the types of services that headspace clients are receiving and to determine whether the approach is making a difference to their mental health and wellbeing. Importantly, a process to obtain follow-up data 3 months after young people have received their last service was implemented several months after the initial implementation of the new minimum dataset, and these outcome data will be available for analysis early in 2014. Such analyses, and other evaluation efforts, are required to determine whether headspace is delivering on the aims of this innovative initiative.

Competing interests: We are all employed by or directly involved with headspace National Youth Mental Health Foundation.

Received 24 Sep 2013, accepted 28 Nov 2013.

1 McGorry PD, Tanti C, Stokes R, et al. headspace: Australia's National Youth Mental Health Foundation - where young minds come first. Med J Aust 2007; 187 (7 Suppl): S68-S70.

2 Slade T, Johnston A, Teesson M, et al. The mental health of Australians 2. Report on the 2007 National Survey of Mental Health and Wellbeing. Canberra: Australian Department of Health and Ageing, 2009.

3 Gibb SJ, Fergusson DM, Horwood LJ. Burden of psychiatric disorder in young adulthood and life outcomes at age 30

Br J Psychiatry 2010; 197: 122-127.

4 Merikangas KR, He JP, Burstein M, et al. Lifetime prevalence of mental disorders in US adolescents: results from the National Comorbidity Survey Replication-Adolescent
Supplement (NCS-A). J Am Acad Child Adolesc Psychiatry 2010; 49: 980-989.

5 Kessler RC, Amminger GP, Aguilar-Gaxiola S, et al. Age of onset of mental disorders: a review of recent literature. Curr Opin Psychiatry 2007; 20: 359-364.

6 McGorry PD. The specialist youth mental health model: strengthening the weakest link in the public mental health system. Med J Aust 2007; 187 (7 Suppl): S53-S56.

7 Rickwood DJ, Van Dyke N, Telford N. Innovation in youth mental health services in Australia: common characteristics across the first headspace centres. Early Interv Psychiatry 2013; Jul 4

[Epub ahead of print]. doi: 10.1111/eip.12071.

8 Gulliver A, Griffiths KM, Christensen H. Perceived barriers and facilitators to mental health helpseeking in young people: a systematic review. BMC Psychiatry 2010; 10: 113.

9 Rickwood DJ, Deane FP, Wilson CJ. When and how do young people seek professional help for mental health problems? Med J Aust 2007; 187 (7 Suppl): S35-S39.

10 Kessler RC, Andrews G, Colpe LJ, et al. Short screening scales to monitor population prevalences and trends in non-specific psychological distress. Psychol Med 2002; 32: 959-976.

11 McGorry PD, Purcell R, Hickie IB, et al. Clinical staging: a heuristic model for psychiatry and youth mental health. Med J Aust 2007; 187 (7 Suppl): S40-S42.

12 Australian Bureau of Statistics. Mental health and wellbeing: profile of adults, Australia, 1997. Canberra: ABS, 1998.

(ABS Cat. No. 4326.0.)

13 Goldman HH, Skodol AE, Lave TR. Revising Axis V for DSM-IV: a review of measures of social functioning. Am J Psychiatry 1992; 149: 1148-1156.

14 Australian Institute of Health and Welfare. Young Australians: their health and wellbeing 2011. Canberra: AlHW, 2011. (AlHW Cat. No. PHE 140.)

15 Australian Bureau of Statistics. Estimates of Aboriginal and Torres Strait Islander Australians, June 2011. Canberra: ABS, 2013. (ABS Cat. No. 3238.0.55.001.)

16 Australian Bureau of Statistics. Migration, Australia, 2007-08. Canberra: ABS, 2009. (ABS Cat. No. 3412.0.)

17 Australian Bureau of Statistics. Reflecting a nation: stories from the 2011 census. Canberra: ABS, 2013. (ABS Cat. No. 2071.0.)

18 Australian Bureau of Statistics. Population estimates by remoteness area, 2011 to 2012. Canberra: ABS, 2013. (ABS Cat. No. 3218.0.)

19 Australian Bureau of Statistics. 2011 Census of population and housing: estimating homelessness, 2011 . Table 8 homeless operational groups and other marginal housing, number of persons-by selected characteristics2011. Canberra: ABS, 2012. (ABS Cat. No. 2049.0.)

20 COAG Reform Council. Education in Australia 2012: five years of performance. Sydney: COAG Reform Council, 2013.

21 Australian Department of Health and Ageing Promotion, prevention and early intervention for mental health: a monograph. Canberra: Australian Government, 2000.

22 Scott J, Fowler D, McGorry P, et al. Adolescents and young adults who are not in employment education, or training. BMJ 2013; 347: 55270.

23 Carbone S, Rickwood D, Tanti C. Workforce shortages and their impact on Australian youth mental health service reform. Advances in Mental Health 2011; 10: 92-97.

24 Kessler RC, Merikangas KR, Berglund P, et al. Mild disorders should not be eliminated from the DSM-V. Arch Gen Psychiatry 2003; 60: 